\title{
Constructions of bipartite graphs from finite geometries
}

\author{
Keith E. Mellinger*and Dhruv Mubayi ${ }^{\dagger}$
}

May 10, 2005

\begin{abstract}
We construct an incidence structure using certain points and lines in finite projective spaces. The structural properties of the associated bipartite incidence graphs are analyzed. These $n \times n$ bipartite graphs provide constructions of $C_{6}$-free graphs with $\Omega\left(n^{4 / 3}\right)$ edges, $C_{10}$-free graphs with $\Omega\left(n^{6 / 5}\right)$ edges, and $\Theta(7,7,7)$-free graphs with $\Omega\left(n^{8 / 7}\right)$ edges. Each of these bounds is sharp in order of magnitude.
\end{abstract}

Keywords: Bipartite Turán problem, finite geometry, even cycles

\section{Introduction}

One of the basic problems in extremal graph theory can be stated as follows. Given a fixed graph $F$, determine $\operatorname{ex}(n, F)$, the maximum number of edges in an $n$ vertex graph containing no copy of $F$ as a subgraph. The parameter $\operatorname{ex}(n, F)$ is sometimes called the Turán number of $F$.

The Erdős-Simonovits-Stone Theorem [7, 8] determines ex $(n, F)$ asymptotically except when $\chi(F)=2$, or $F$ is bipartite; in this case all it says is that $\operatorname{ex}(n, F)=o\left(n^{2}\right)$. Among bipartite graphs, there are two important classes of graphs for which the Turán problem remains open: complete bipartite graphs and even cycles. It is fair to say that these are two of the

${ }^{*}$ Research supported in part by an NSF VIGRE grant

${ }^{\dagger}$ Research supported in part by NSF grants DMS 9970325 and DMS 0400812 
major open problems in extremal graph theory. In this paper, we are mainly concerned with lower bounds for $\operatorname{ex}\left(n, C_{2 t}\right)$.

Reiman [14] observed that ex $\left(n, C_{4}\right) \leq n^{3 / 2} / 2+n / 4$, and Bondy and Simonovits [2] showed more generally that $\operatorname{ex}\left(n, C_{2 t}\right) \leq 100 t n^{1+1 / t}$. Recently, Verstraëte [15] improved the constant to $8(t-1)$. Constructions of $n$-vertex $C_{2 t}$-free graphs with $\Omega\left(n^{1+1 / t}\right)$ edges are known only for $t=2$ (Brown [3] and Erdős-Rényi [6]), 3, and 5. For the latter two cases, independent constructions have been given by Benson [1], Wenger [16], and Lazebnik-UstimenkoWoldar [11].

The first author [13] recently provided a construction that yields ex $\left(n, C_{6}\right)=$ $\Omega\left(n^{4 / 3}\right)$. This arose from a triangle-free set of lines in projective 3 -space, and resulted in an $m$ by $m$ bipartite graph with girth 8 and $(1+o(1)) m^{4 / 3}$ edges. We could not prove that it is not isomorphic to one of the previous bipartite constructions in [11]. Certainly the geometric language in which the construction is phrased, and the proof that the construction works, are both new. In particular, it would be interesting to determine the relationship between this construction and known generalized quadrangles.

In this paper, we generalize the construction from [13] to $t$-dimensional projective spaces. This gives us an $m$ by $m$ bipartite graph $B_{t}$ with $(1+$ $o(1)) m^{1+1 / t}$ edges for infinitely many $m$. Our first result is to prove that the graph $B_{5}$ contains no cycle of length 10 . Our construction does not yield the densest known $m$ vertex graphs without $C_{10}$.

Our hope was that the same approach would yield $\operatorname{ex}\left(n, C_{2 t}\right)=\Omega\left(n^{1+1 / t}\right)$ for other values of $t$, in particular, for $t=4$ (which is somewhat of a notorious open case). Unfortunately, this was not the case, and the closest we got was for $t=7$. The graph $B_{7}$ does contain copies of $C_{14}$, but we prove that every two vertices of $B_{7}$ have at most two pairwise internally disjoint paths of length 7 between them (note that $C_{14}$ is a graph consisting of exactly two internally disjoint paths of length 7 with the same endpoints). Consequently, we obtain the new result

$$
\operatorname{ex}(n, \Theta(7,7,7))=\Omega\left(n^{8 / 7}\right),
$$

where the theta graph $\Theta(7,7,7)$ is the graph consisting of three pairwise internally disjoint paths of length 7 with the same endpoints. Moreover, the exponent $8 / 7$ is sharp, as shown by a result of Faudree and Simonovits [9].

It is worth remarking that determining the order of magnitude of the Turán number of any bipartite graph (with a cycle) is nontrivial. For ex- 
ample, $\operatorname{ex}\left(n, Q_{3}\right)$, where $Q_{3}$ is the 3-dimensional cube, is a well-known open problem.

Our final result concerns an asymmetric bipartite version of the Turán problem. Let $f(n, m)$ denote the maximum number of edges in an $n$ by $m$ bipartite graph $(n \geq m)$ with girth at least 8 . Erdös conjectured that $f(n, m)=O(n)$ for $m=O\left(n^{2 / 3}\right)$, and this was disproved by de Caen and Székely [4] who proved that $f(n, m) \geq n^{58 / 57+o(1)}$ for $m=(1+o(1)) n^{2 / 3}$. The best known lower bound due to Lazebnik, Ustimenko and Woldar [12] is $\Omega\left(n^{16 / 15}\right)$. In the other direction, an upper bound of de Caen and Székely [4] is $O\left(n^{10 / 9}\right)$. A slight variation of our construction produces a bipartite graph that matches the lower bound in [12]. We emphasize here that our proof uses only basic facts about projective geometries, and is extremely short.

The paper is organized as follows. Section 2 contains the main construction used throughout. In section 3 , we give a quick proof of the result in [13] for $t=3$ and demonstrate the pitfalls of the $t=4$ case by showing that our graphs do contain many copies of $C_{8}$. In section 4 we prove the $t=5$ case, and in section 5 we prove (1). We end in section 6 by modifying our graphs a little to obtain the claimed lower bound for $f(n, m)$, namely that $f(n, m) \geq \Omega\left(n^{16 / 15}\right)$ for $m=(1+o(1)) n^{2 / 3}$.

\section{The arc construction}

For $t \geq 2$ and $q$ a prime power, let $\Sigma=P G(t, q)$ represent the $t$-dimensional projective space over the finite field $G F(q)$. Let $\Sigma_{0}$ represent a hyperplane of $\Sigma$, that is, an isomorphic copy of $P G(t-1, q)$ inside $\Sigma$.

An $\operatorname{arc}$ of $\Sigma_{0}$ is a collection of points of $\Sigma_{0}$ such that no $t$ points are in a $(t-2)$-dimensional subspace of $\Sigma_{0}$. For $q \geq t$, a standard construction of an arc with $q+1$ points is given by the normal rational curve as described in [10]. Letting $\Sigma_{0}$ be the set of points whose first homogeneous coordinate is 0 , this arc consists of the points

$$
\left\{\left(0,1, x, x^{2}, x^{3}, \ldots, x^{t}\right): x \in G F(q)\right\} \cup\{(0,0,0, \ldots, 0,1)\} .
$$

Construction: Let $\mathcal{A}$ be the normal rational curve in $\Sigma_{0}$. Let $H(t, q)$ denote the bipartite graph with parts $P$ and $L$. The set $P$ consists of the points of $\Sigma \backslash \Sigma_{0}$ and $L$ consists of all the lines of $\Sigma$ not in $\Sigma_{0}$ that meet the hyperplane 
$\Sigma_{0}$ in a point of $\mathcal{A}$. The vertex $p \in P$ is adjacent to $l \in L$ if the point $p$ lies on the line $l$ in $\Sigma$. An easy counting argument shows that $|P|=q^{t}$ and $|L|=q^{t-1}(q+1)$. Moreover, each vertex in $P$ has degree $|\mathcal{A}|=q+1$ and each vertex in $L$ has degree $q$. Letting $m=q^{t}$, we see that $H(t, q)$ has part sizes $m$ and $m+m^{1-1 / t}$, and $m+m^{1+1 / t}$ edges.

Remark: Deleting $m^{1-1 / t}$ vertices from $L$ results in an $m \times m$ bipartite graph with $m^{1+1 / t}$ edges. For every $\epsilon>0$, there is an $n_{0}$ such that for $n>n_{0}$, the interval $(n, n+\epsilon n)$ contains a prime. Consequently, for fixed $t$, the graphs $H(t, q)$ produce a family of $m$ by $m$ bipartite graphs with $(1+o(1)) m^{1+1 / t}$ edges.

\section{6-cycles and 8-cycles}

In this section, we analyze the graphs $H(3, q)$ and $H(4, q)$. When $t=3$, the arc $\mathcal{A}$ is a collection of $q+1$ points of $\operatorname{PG}(2, q)$, no three collinear. The construction in this case was described in [13] where the associated graphs were used to construct low-density parity check codes. We repeat the argument here instead focusing on the associated graph.

Theorem 3.1 The graph $H(3, q)$ has girth 8.

Proof: First note that $H(3, q)$ contains no 4-cycles, since every two lines of $L$ have at most one point in common. Now, for contradiction, suppose that the lines $l_{1}, l_{2}$, and $l_{3}$ of $L$ form a triangle. Hence, any two of the three lines meet in a point of $\Sigma \backslash \Sigma_{0}$. Then these three lines meet the hyperplane $\Sigma_{0}$ in three distinct points $A_{1}, A_{2}$, and $A_{3}$ of $\mathcal{A}$. Since the lines are necessarily coplanar, these three points meet on the line determined by the intersection of $\Sigma_{0}$ and the plane containing the triangle. Hence, the three points $A_{1}, A_{2}$, and $A_{3}$ are collinear contradicting the definition of arc.

To show the girth is exactly 8, we exhibit an 8-cycle. Denoting projective points by their homogeneous coordinates, and lines with a pair of linear equations on the coordinates $(w, x, y, z)$, our desired 8-cycle is given by $(1,0,0,0) \rightarrow\{y=z=0\} \rightarrow(1,1,0,0) \rightarrow\{w=x, y=0\} \rightarrow(1,1,0,1) \rightarrow$ $\{w=z, y=0\} \rightarrow(1,0,0,1) \rightarrow\{x=y=0\} \rightarrow(1,0,0,0)$.

When $t=4$, the $\operatorname{arc} \mathcal{A}$ is a collection of $q+1$ points of $P G(3, q)$, no four coplanar. As mentioned in the introduction, $H(4, q)$ does in fact contain 8-cycles. 
Lemma 3.2 Let $P_{1}, P_{2}, P_{3}$, and $P_{4}$ be four distinct points of $\Sigma \backslash \Sigma_{0}$ which form a quadrilateral with the lines of $L$. Then the four points are coplanar.

Proof: For a contradiction, suppose that the four points are not coplanar. First note that the lines of the quadrilateral necessarily meet the arc in 4 distinct points since if two lines passed through the same point of the arc, then the four points $P_{i}$ would all lie in a common plane. Now, the four points $P_{i}$ span a solid of $\Sigma$ which necessarily meets the hyperplane $\Sigma_{0}$ in a projective plane. The lines determining the quadrilateral are all contained in this solid and meet $\Sigma_{0}$ in 4 distinct points of the arc. But these four points are then coplanar contradicting the definition of arc.

By Lemma 3.2, all 8-cycles of the associated graph arise from quadrilaterals lying in a plane. One might hope that the number of these is relatively small, allowing for some clever deletion procedure that destroys all these cycles, but this is not the case. In fact, given any two distinct points $A, A^{\prime}$ on $\mathcal{A}$, choose any plane $\pi$ of $\Sigma$, not in $\Sigma_{0}$ and containing the points $A$ and $A^{\prime}$. Any two lines of $\pi$ through $A$, together with any two lines of $\pi$ through $A^{\prime}$ will form a quadrilateral. In $H(4, q)$ this is an 8-cycle. Moreover, as no 3 points of $\mathcal{A}$ are collinear, the 8-cycles so obtained are pairwise distinct. Standard counting shows that the number of such quadrilaterals is $\left(\begin{array}{c}q+1 \\ 2\end{array}\right)\left(\begin{array}{c}q \\ 2\end{array}\right)^{2} q^{2}$. There appears to be no way to delete a small number of edges from $H(4, q)$ to destroy all these copies of 8-cycles.

\section{No 10-cycles}

When $t=5$, we are using an arc of $\Sigma_{0} \cong P G(4, q)$. Our incidence structure contains $q^{4}(q+1)$ lines and $q^{5}$ points. In this case we will show that $H(5, q)$ contains no 10 -cycles, however, it will certainly contain 8-cycles. The same argument given earlier shows that $H(5, q)$ has no $C_{6}$ which implies that $H(5, q)$ has girth 8 .

Theorem 4.1 The line set $L$ does not contain 5 lines forming a pentagon.

Proof: Let $\mathcal{P}=\left\{P_{1}, P_{2}, P_{3}, P_{4}, P_{5}\right\}$ be 5 points of $\Sigma \backslash \Sigma_{0}$ (in cyclic order) which form a pentagon. Moreover, let $l_{i} \in L$ be the line joining $P_{i}$ and $P_{i+1}$, where indices are taken modulo 5. We look at cases based on the dimension of the space spanned by these five points. Clearly this dimension is greater than 1. 
- Case 1: Suppose that the five points are all coplanar. Then a straight forward pigeonhole argument shows that the five lines $l_{i}$ must meet $\mathcal{A}$ in at least 3 points which are necessarily collinear. This contradicts the definition of $\mathcal{A}$.

- Case 2: Suppose that the five points span a solid. We claim that the five lines $l_{i}$ must meet $\mathcal{A}$ in at least 4 points. If not, then two points of $\mathcal{A}$, say $A_{1}$ and $A_{2}$ would each be incident with two different lines. Without loss of generality, assume that $l_{1}$ and $l_{3}$ are incident with $A_{1}$ and $l_{2}$ and $l_{4}$ are incident with $A_{2}$. Then the plane $\pi_{1}$ determined by $l_{1}$ and $l_{3}$ and the plane $\pi_{2}$ determined by $l_{2}$ and $l_{4}$ both contain the three noncollinear points $P_{2}, P_{3}, P_{4}$. Consequently, $\pi_{1}=\pi_{2}$. But this immediately implies that $\mathcal{P}$ is in a plane which contradicts the assumptions of Case 2. Therefore, the five lines $l_{i}$ meet $\mathcal{A}$ in at least 4 points. The solid of $\Sigma$ determined by $\mathcal{P}$ meets $\Sigma_{0}$ in a plane. But this plane contains 4 points of $\mathcal{A}$ by the argument above contradicting the definition of $\mathcal{A}$.

- Case 3: Finally, suppose that the five points of the pentagon span a subspace of dimension 4 . Then, we claim that the lines $l_{i}$ must meet $\mathcal{A}$ in 5 distinct points. If not, then the pentagon contains two lines $l_{i}$ and $l_{j},|i-j|>1$, which are coplanar, and hence four of its points are all in a common plane. This means that $\mathcal{P}$ can span at most a solid, contradicting the assumption of Case 3 . So the lines $l_{i}$ meet $\mathcal{A}$ in 5 distinct points. But the intersection of $\Sigma_{0}$ with the space spanned by $\mathcal{P}$ forms a solid. Hence, these five points of the arc all lie in a common solid. This again contradicts the definition $\mathcal{A}$.

The argument to prove Lemma 3.2 shows that if a quadrilateral is formed by the lines of $L$, then the lines of the quadrilateral are necessarily coplanar. Hence the girth of $H(5, q)$ is 8 , but it contains no 10-cycles.

\section{No $\Theta(7,7,7)$}

When $t=7$, we are working in $P G(7, q)$ with an arc of $P G(6, q)$. Let $\mathcal{P}=\left\{P_{1}, \ldots P_{7}\right\}$ be seven points of $\Sigma \backslash \Sigma_{0}$ (in cyclic order) which form a 7-sided figure, a septagon. Moreover, let $l_{i} \in L$ be the line joining points $P_{i}$ 
and $P_{i+1}$, where indices are taken modulo 7 . Note that the $l_{i}$ s are distinct and hence every 3 consecutive points of $\mathcal{P}$ are noncollinear.

Our proof in this case is considerably more involved and will be aided with some new notation. Let $A_{i}$ be the point of the $\operatorname{arc} \mathcal{A}$ which is incident with $l_{i}$ (note that $A_{i}=A_{j}$ for $i \neq j$ is possible). Let $d$ be the dimension of the subspace of $\Sigma$ spanned by the points of $\mathcal{P}$, and let $a$ be the number of distinct $A_{i}$ s.

We first point out a relationship between $a$ and $d$. Note that the dimension of $\Sigma_{0} \cap\langle\mathcal{P}\rangle=d-1$ since $\Sigma_{0}$ is a hyperplane and $\mathcal{P}$ contains points outside of $\Sigma_{0}$. Also, recall that no $d+1$ points of $\mathcal{A}$ lie in a subspace of dimension $d-1$. Since each $A_{i}$ lies in $\Sigma_{0} \cap\langle\mathcal{P}\rangle$, we obtain

$$
a \leq d
$$

We will use this relationship throughout the proof of the main theorem. We first note that $d>1$. Otherwise all $l_{i}$ are equal which is clearly a contradiction. Also, note that $\bigcup_{i}\left\{A_{i}\right\}$ induces a coloring of the lines forming our septagon, where the color $c(l)$ of the line $l$ is the unique $k$ for which $A_{k} \in l$. Note that $c\left(l_{i}\right) \neq c\left(l_{i+1}\right)$ for each $i$, since two distinct lines share at most one point. We write $\left[c_{1}, c_{2}, \ldots, c_{7}\right]$ for this sequence of colors, where $c_{i}=c\left(l_{i}\right)$ for each $i$.

Lemma 5.1 Let $P_{i}, P_{i+1}, P_{j} \in \mathcal{P}$ with $j \notin\{i-1, i+2\}$. If $c\left(l_{i}\right)=c\left(l_{k}\right)$ for $k=j-1$ or $k=j$, then $P_{i}, P_{i+1}$ and $P_{j}$ are noncollinear.

Proof: For contradiction, suppose $P_{i}, P_{i+1}$ and $P_{j}$ are collinear. Then the lines $l_{i}$ and $l_{k}$ both contain $P_{j}$ and $A_{i} \in \mathcal{A}$. This implies that $l_{i}=l_{k}$ contradicting the fact that the $l_{i}$ s are all distinct.

Lemma 5.2 Suppose that the set of points in $\mathcal{P}$ form a septagon. Then the colors of the lines $l_{i}$ follow the pattern $[x, y, x, z, x, y, z]$ for three distinct colors $x, y$, and $z$.

Proof: We prove the result by considering cases based on the value of $a$, the number of points of $\mathcal{A}$ which lie on the lines forming our septagon. In each case, we consider the various configurations of the points of $\mathcal{P}$, and we obtain a contradiction with (2). 
- Case $a=2$ : In this case, a straightforward pigeon hole argument shows that $l_{i}=l_{i+1}$ for some $i$. This is a contradiction.

- Case $a=3$ : Note that the 3 points of the arc induce a 3-coloring on the lines of $L$. A pigeon hole argument shows that no color can appear more than three times. Hence, up to equivalence, the induced coloring is of the type $[x, y, x, y, x, y, z],[x, y, x, z, x, z, y]$, or $[x, y, x, z, x, y, z]$ for three distinct colors $x, y$, and $z$. We prove that the first two cases are impossible. For the following, let $\pi\left(l_{i}, l_{j}\right)$ represent the plane generated by the two concurrent lines $l_{i}$ and $l_{j}$.

- If the coloring follows the pattern $[x, y, x, y, x, y, z]$, then $\pi\left(l_{1}, l_{3}\right)=$ $\pi\left(l_{2}, l_{4}\right)$ since these planes share the three noncollinear points $P_{2}, P_{3}, P_{4}$. Similarly, $\pi\left(l_{2}, l_{4}\right)=\pi\left(l_{3}, l_{5}\right)=\pi\left(l_{4}, l_{6}\right)$. Hence, all 7 points of $\mathcal{P}$ are in a common plane which means $d=2$, a contradiction.

- Now suppose the coloring of $L$ follows the pattern $[x, y, x, z, x, z, y]$. Then let $\pi_{1}=\pi\left(l_{1}, l_{3}\right)=\pi\left(l_{2}, l_{7}\right)$, and $\pi_{2}=\pi\left(l_{4}, l_{6}\right)$. Now, $A_{1}$ (the point of $\mathcal{A}$ on $l_{1}, l_{3}$, and $l_{5}$ ) is in $\pi_{1}$ and also in $\pi_{2}$. So, either $\pi_{1}=\pi_{2}$ in which case we are finished since then $d=2$, or $\pi_{1}$ meets $\pi_{2}$ in a line (since both planes contain $P_{4}$ and $P_{7}$ ) which passes through the point $A_{1}$. But then this line $\pi_{1} \cap \pi_{2}$ forms a pentagon with the lines $l_{1}, l_{2}, l_{3}$ and $l_{7}$ which is a contradiction with Theorem 4.1.

- Case $a=4$ : We consider the various coloring patterns of the $l_{i} \mathrm{~s}$.

- Suppose we have one color appearing three times, one color appearing two times and two colors appearing one time each. Let $x$ represent the color appearing three times. Then the three lines with color $x$ all share the same point on $\mathcal{A}$ and so generate at most a solid. If they generate a plane, then we are finished since then $d \leq 3$. So assume they generate a solid.

We claim that the pattern $[x, y, x, y, x]$ cannot appear as a subpattern in any coloring. If so, then the plane generated by the lines colored with $y$ would necessarily contain the three lines colored with $x$ forcing these lines to be coplanar, a contradiction. Hence, the only possible coloring patterns are $[x, y, x, z, x, y, w]$ 
or $[x, y, x, z, x, w, y]$ (for example, $[x, y, x, z, x, z, w]$ is equivalent to $[x, y, x, z, x, w, y]$ by interchanging $y$ and $z$ and reversing the orientation). In the first case, the plane generated by the lines colored with $y$ shares the three points $P_{2}, P_{3}$ and $P_{6}$ with the solid generated by the lines colored with $x$. By Lemma 5.1 these three points are noncollinear. In the second case, the two planes share the three noncollinear points $P_{1}, P_{2}$ and $P_{3}$. Consequently, in either case, $\mathcal{P}$ lies in the solid generated by the lines colored with $x$, implying $d=3$, a contradiction.

- Now consider the possibility of 3 distinct colors $x, y, z$ each appearing twice, and one additional color appearing only once. For $u \in\{x, y, z\}$, let $\pi_{u}$ be the plane determined by the two lines colored with $u$. We look at the union of four lines which represent two colors, say $x$ and $y$. If these two colors appear in the subpattern $[x, y, x, y]$, then the four lines together generate a plane. Since $\pi_{z}$ meets $\pi_{x}=\pi_{y}$ in a line. It follows that the set $\mathcal{P}$ spans at most a solid, contradicting (2).

We may therefore assume that no two of the repeated colors give the pattern $[p, q, p, q]$. From this, a short case analysis shows that for some pair of repeated colors, say $x, y$, the planes $\pi_{x}$ and $\pi_{y}$ meet in two points of $\mathcal{P}$, and therefore span at most a solid. Moreover, the possible patterns for the seven colors are [xy**xy*], [xy**yx*], and $[x y x * y * *]$. Now $\pi_{z}$ has three points in common with $S$. A short case analysis, together with Lemma 5.1, shows that for each pattern above, these three points are noncollinear. Therefore $\mathcal{P}$ lies within $S$, contradicting (2).

- Case $a=5$ : First suppose that one color appears three times and all the remaining colors appear exactly once. Then, the three lines sharing the same color generate at most a solid and only one point of $\mathcal{P}$ is outside this solid. Hence, the points of $\mathcal{P}$ generate at most a 4-space, contradicting (2).

The only other possibility is that two distinct colors appear twice each and three others colors appear once each. The union of the lines from the two repeating colors generate at most a 4 -space. If these lines generate a solid, then at most one point of $\mathcal{P}$ can lie outside this solid. If these lines generate a 4 -space, then no points of $\mathcal{P}$ can lie outside of 
this 4-space. In either case, $d \leq 4$, a contradiction.

- Case $a=6$ : In this case, only one color appears twice and all other colors appear exactly once. The lines having the same color generate a plane. At most three points of $\mathcal{P}$ lies outside this plane. It follows immediately that the points of $\mathcal{P}$ generate at most a 5 -space, a contradiction.

Theorem 5.3 The graph $H(7, q)$ contains no copy of $\Theta(7,7,7)$.

Proof: Suppose the statement is false. Then there exist two septagons in $\Sigma \backslash \Sigma_{0}$ which share exactly 4 consecutive points and 4 consecutive lines. Let $C_{1}$ be a septagon formed by the lines $l_{1}, l_{2}, l_{3}, l_{4}, l_{5}, l_{6}$ and $l_{7}$ and let $C_{2}$ be a second septagon formed by the lines $l_{1}, l_{2}, l_{3}, l_{4}, l_{5}^{\prime}, l_{6}^{\prime}$ and $l_{7}^{\prime}$. First note that we obtain a third septagon $C_{3}$ formed by the lines $l_{7}^{\prime}, l_{6}^{\prime}, l_{5}^{\prime}, l_{4}, l_{5}, l_{6}$ and $l_{7}$. By Lemma 5.2, the colors of each of these line sequences must follow a pattern of the form $[x, y, x, z, x, y, z]$. Without loss of generality, we can assume that two of the lines $l_{1}, l_{2}, l_{3}$ and $l_{4}$ are colored with the same color, say $x$, which appears 3 times in each of the cycles involving $l_{1}, l_{2}, l_{3}$ and $l_{4}$. We have three cases.

- Suppose $l_{1}, l_{2}, l_{3}$ and $l_{4}$ are colored in the pattern $[x, y, z, x]$. Then $l_{5}, l_{6}$ and $l_{7}$ cannot be colored with only the colors $y$ and $z$, implying $c\left(l_{6}\right)=$ $x$. Similarly, $c\left(l_{6}^{\prime}\right)=x$. Now consider the sequences $l_{2}, l_{1}, l_{7}, l_{6}$ and $l_{2}, l_{1}, l_{7}^{\prime}, l_{6}^{\prime}$. Each is colored in the pattern $[y, x, *, x]$. In order to avoid the unallowable pattern $[y, x, y, x]$, we must have $c\left(l_{7}\right)=c\left(l_{7}^{\prime}\right)=z$. Consequently, the two adjacent lines, $l_{7}$ and $l_{7}^{\prime}$ in $C_{3}$, have the same color, a contradiction.

- Suppose $l_{1}, l_{2}, l_{3}$ and $l_{4}$ are colored in the pattern $[x, y, x, z]$. Consider the sequences $l_{3}, l_{2}, l_{1}, l_{7}$ and $l_{3}, l_{2}, l_{1}, l_{7}^{\prime}$. Each is colored in the pattern $[x, y, x, *]$. Then $l_{7}$ and $l_{7}^{\prime}$ must each be colored with $z$ to avoid an $[x, y, x, y]$ pattern in $C_{1}$ or $C_{2}$. Again, this gives two adjacent lines in $C_{3}$ with the same color, a contradiction. 
- Suppose $l_{1}, l_{2}, l_{3}$ and $l_{4}$ are colored in the pattern $[z, x, y, x]$. Then $c\left(l_{5}\right)=c\left(l_{5}^{\prime}\right)=z$ by looking at the sequences $l_{2}, l_{3}, l_{4}, l_{5}$ and $l_{2}, l_{3}, l_{4}, l_{5}^{\prime}$ and avoiding the $[x, y, x, y]$ pattern. But then $l_{6}$ and $l_{7}$ must be colored as $[x, y]$ or $[y, x]$ since both are adjacent to a line colored with $z$. The same argument applies to $l_{6}^{\prime}$ and $l_{7}^{\prime}$. But $l_{7}$ and $l_{7}^{\prime}$ cannot both have the same color since they are adjacent in $C_{3}$. Hence, without loss of generality, assume that $c\left(l_{7}\right)=x$ and $c\left(l_{7}^{\prime}\right)=y$. This implies $l\left(c_{6}\right)=$ $y$ and so $c\left(l_{6}^{\prime}\right)=x$ producing an $[x, y, x, y]$ pattern in $C_{3}$, again a contradiction.

In all cases, we get a contradiction. Therefore, the graph $H(7, q)$ cannot contain 2 vertices with three distinct (and pairwise internally disjoint) paths of length 7 between them.

\section{Other graphs from caps}

In this section we obtain the claimed lower bound on $f(n, m)$. Our approach is to use caps of the hyperplane $\Sigma_{0}$ in higher dimensional spaces to obtain bipartite graphs with unequal part sizes.

Theorem 6.1 Let $q$ be a prime power. Then there is a bipartite graph with part sizes $q^{11}\left(q^{2}+1\right)^{2}$ and $q^{10}$, girth 8 , and $q^{12}\left(q^{2}+1\right)^{2}$ edges.

Proof: We modify our construction of $H(10, q)$. Instead of using an arc, we use a cap, namely, a set of points, no three collinear. In [5] it is shown that there exists a cap $\mathcal{C}$ of $P G(9, q)$ consisting of $q^{2}\left(q^{2}+1\right)^{2}$ points. Embed $\Sigma_{0} \cong P G(9, q)$ containing $\mathcal{C}$ into $\Sigma=P G(10, q)$. Form the bipartite graph $H^{\prime}(10, q)$ with parts $P$ and $L$, where $P$ consists of the point in $\Sigma \backslash \Sigma_{0}$ and $L$ consists of lines not contained in $\Sigma_{0}$ that meet $\mathcal{C}$. Adjacency is again defined by incidence in the geometry.

Easy counting shows that the part sizes of $H^{\prime}(10, q)$ are $q^{10}$ and $q^{11}\left(q^{2}+\right.$ $1)^{2}$, and the number of edges is $q^{12}\left(q^{2}+1\right)^{2}$. The proof of Theorem 3.1 applies to show that $H^{\prime}(10, q)$ has girth at least 8 .

Corollary $6.2 f(n, m) \geq(1+o(1)) n^{16 / 15}$ for $m=(1+o(1)) n^{2 / 3}$. 


\section{Acknowledgments}

The authors are grateful to the referees for their helpful comments.

\section{References}

[1] C. T. Benson, Minimal regular graphs of girths eight and twelve, Canad. J. Math. 18 (1966) 1091-1094.

[2] A. J. Bondy, M. Simonovits, Cycles of even length in graphs, J. Combinatorial Theory Ser. B 16 (1974), 97-105.

[3] W. G. Brown, On graphs that do not contain a Thomsen graph, Canad. Math. Bull. 9 (1966) 281-289.

[4] D. de Caen, D, L. Székely, The maximum size of 4- and 6-cycle free bipartite graphs on $m, n$ vertices. Sets, graphs and numbers (Budapest, 1991), 135-142, Colloq. Math. Soc. Jnos Bolyai, 60, North-Holland, Amsterdam, 1992.

[5] Y. Edel and J. Bierbrauer, Recursive constructions for large caps. Bull. Belg. Math. Soc. 6 (1999), no. 2, 249-258.

[6] P. Erdős, A. Rényi, On a problem in the theory of graphs. (Hungarian) Magyar Tud. Akad. Mat. Kutat Int. Kzl. 7 (1962) 623-641.

[7] P. Erdős, M. Simonovits, A limit theorem in graph theory, Studia Sci. Math. Hungar. 1 (1966) 51-57.

[8] P. Erdős, A. H. Stone, On the structure of linear graphs. Bull. Amer. Math. Soc. 52 (1946) 1087-1091.

[9] R. J. Faudree, M. Simonovits, On a class of degenerate extremal graph problems. Combinatorica 3 (1983), no. 1, 83-93

[10] J.W.P. Hirschfeld and J. Thas, General Galois Gemetries, Oxford University Press.

[11] F. Lazebnik and V. A. Ustimenko, Explicit construction of graphs with an arbitrary large girth and of large size. Discrete Appl. Math. 60 (1995), no. 1-3, 275-284. 
[12] F. Lazebnik, V. A. Ustimenko and A. J. Woldar, New constructions of bipartite graphs on $m, n$ vertices with many edges and without small cycles. J. Combin. Theory Ser. B 61 (1994), no. 1, 111-117.

[13] K. E. Mellinger, LDPC codes from triangle-free line sets, Designs, Codes, and Cryptog., 32 (2004), no. 1-3, 341-350.

[14] I. Reiman, Über ein Problem von K. Zarankiewicz (German), Acta. Math. Acad. Sci. Hungar. 9 (1958) 269-273.

[15] J. Verstraëte, On arithmetic progressions of cycle lengths in graphs, Combin. Probab. Comput. 9 (2000), no. 4, 369-373.

[16] R. Wenger, Extremal graphs with no $C^{4}$ 's, $C^{6}$ 's, or $C^{10}$ 's, J. Combin. Theory Ser. B 52 (1991), no. 1, 113-116.

Keith E. Mellinger

Department of Mathematics

University of Mary Washington

1301 College Avenue

Fredericksburg, VA 22401

kmelling@mwc.edu

Dhruv Mubayi

Department of Mathematics, Statistics, and Computer Science (mc 249)

University of Illinois at Chicago

Chicago, IL 60607-7045

mubayi@math . uic.edu 